\title{
Extrapolation of nucleus-nucleus cross section to cosmic ray energies using geometrical model
}

\section{Z. Plebaniak ${ }^{* a}$, T. Wibig ${ }^{a, b}$}

${ }^{a}$ National Centre for Nuclear Research, Astrophysics Division, Cosmic Ray Laboratory, ul. 28 Pułku Strzelców Kaniowskich 69, 90-558 Łódź, Poland, E-mail: zp@ zpk • u . lodz . pl

${ }^{b}$ Faculty of Physics and Applied Informatics, University of Łódź, ul. Pomorska 149/153, 90-236 Łódź, Poland.

\begin{abstract}
Inelastic nucleus-nucleus (N-N) cross section is one of the most important physical parameters of an Extensive Air Shower (EAS) development. Value of this parameter was measured in past with relatively big uncertainty by few ground-based cosmic ray experiments up to $95 \mathrm{TeV}$ in c.m.s. For simulation of EAS development at Ultra High Energy Cosmic Rays (UHECR) it is necessary to extrapolate N-N cross-section using phenomenological models. In this work we present results of calculations of $\mathrm{N}-\mathrm{N}$ cross section using few approaches including some approximations of the exact Glauber theory. For calculation we used new parameterization of the hadronic matter distribution in the geometrical model with "BEL behaviour" of proton. Used obtained by us recently model based on the LHC data up to $8 \mathrm{TeV}$ contains four free energy-dependent parameters. Finally, we present comparison of results of $\mathrm{N}-\mathrm{N}$ cross-section calculations using various methods and their comparison with experimental data.
\end{abstract}

35th International Cosmic Ray Conference - ICRC2017

10-20 July, 2017

Bexco, Busan, Korea

${ }^{*}$ Speaker. 


\section{Introduction}

The development of Extensive Air Showers (EAS) depends on inelastic cross sections involving nuclei: the projectile is in general the single nucleon, but for the initial interaction, the primary cosmic rays particle could also be the heavy nuclei, up to Fe. The target is the atmosphere, so it is always nitrogen, oxygen and sometimes argon. To describe interaction cross sections for very high energies we need the theoretical model to transform the nucleon-nucleon cross section into more complicated complex systems. Fortunately since half of the previous century there exist the model which works very well in this field: the Glauber diffraction theory [1,2]. The incoming plane-wave interacts with the partially absorbing object. The waves effectively describe small momentum transfer collisions and the scattering intensity is cast near the small momentum transfer. The diffractive picture required the wavelength to be small as compared to the dimension of the scattering obstacle.

The model is often called "the optical model" with the analogy to the transmission of light through the gray media [3]. The original Glauber description is more general and it is a deep quantum description of the scattering processes. The Glauber approximation which makes the model working well introduces the eikonal function which incorporated all the phase shifts of all possible scattering processes into one complex function $\Omega$.

\section{Optical model and proton-proton cross section}

Single nucleon-nucleon scattering can be described with the one, in general complex function $\chi$, which depicts phase shift. It is related to the scattering amplitude by the two-dimensional Fourier transform

$$
S(\mathbf{t})=\frac{i}{2 \pi} \int \mathrm{e}^{i t \mathbf{b}}\left(1-\mathrm{e}^{i \chi(b)}\right) d^{2} \mathbf{b}
$$

where $\mathbf{b}$ is the impact parameter.

The inverse transformation

$$
1-\mathrm{e}^{i \chi(b)}=\frac{1}{2 \pi i} \int \mathrm{e}^{-i \mathbf{t b}} S(\mathbf{t}) d^{2} \mathbf{t}
$$

suggest the analogy of the proposed description of the collision to the transmission of the light through the gray medium. The optical interpretation leads to the relation of $\chi$ to the each colliding objects (optical) density:

$$
\chi(b) \sim i \int d^{2} \mathbf{b}^{\prime} \rho_{a}(\mathbf{b}) \rho_{b}\left(\mathbf{b}+\mathbf{b}^{\prime}\right)
$$

In the paper [4] we have shown that the optical model with the properly chosen functional dependence of the nucleon profiles can give the correct description of diffractive elastic scattering cross sections of proton-proton (and antiproton) collisions from low $(\sqrt{s} \sim 10 \mathrm{GeV})$ to LHC energies $(\sqrt{s} \sim 10 \mathrm{TeV})$, but what is more important from the cosmic ray point of view it allows the smooth description of the energy dependence of cross sentions given in the eikonal picture by: 


$$
\begin{gathered}
\sigma_{\text {tot }}=2 \int\left[1-\Re\left(\mathrm{e}^{-i \chi(b)}\right)\right] d^{2} \mathbf{b} \\
\sigma_{\mathrm{el}}=\int\left|1-\mathrm{e}^{-i \chi(b)}\right|^{2} d^{2} \mathbf{b} \\
\sigma_{\text {inel }}=\int\left[1-\left|\mathrm{e}^{-i \chi(b)}\right|^{2}\right] d^{2} \mathbf{b}
\end{gathered}
$$

Essense if this model is proposed distribution of hadron matter density consist four energydependence parameters, described by Eq. 2.5. Energy dependence of all parameters is shown on Fig. 4.

$$
\rho_{h}(\mathbf{r})=\frac{1}{8 \pi}\left(c_{1} m_{1}^{3} e^{-m_{1}|\mathbf{r}|}+c_{2} m_{2}^{3} e^{-m_{2}|\mathbf{r}|}\right) .
$$

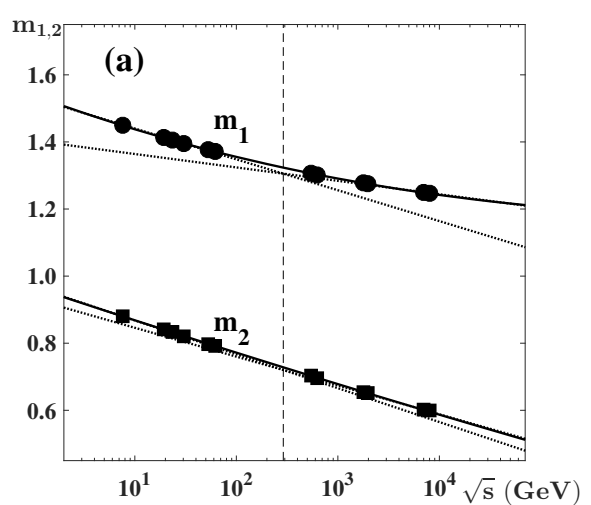

(a) Slopes of two introduced exponential functions $\left(\mathrm{m}_{1}\right.$ and $\left.\mathrm{m}_{2}\right)$

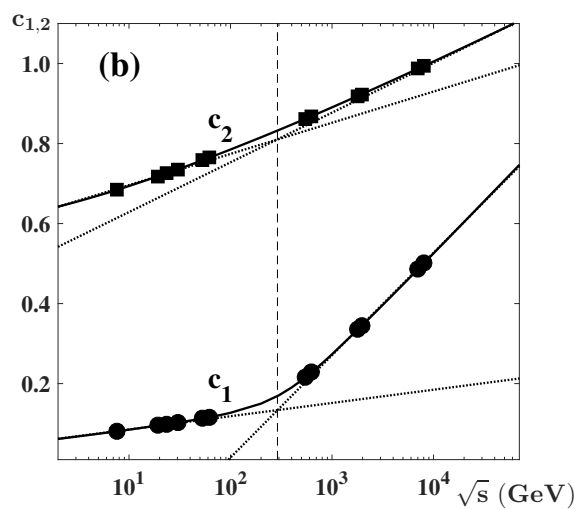

(b) Two different normalization factors $\left(\mathrm{c}_{1}\right.$ and $\left.\mathrm{c}_{2}\right)$

Figure 1: Energy dependence of adjusted parameters in performed p-p optical picture

Introduced distribution allows for calculation of elastic, inelastic an total cross sections According to Eq. 2.5. Results in comparison with experimental data are shown on Fig. 2.

\section{Glauber model for the nucleus case}

The optical model developed further by Czyż and co-workers [5, 6, 7, 8, 9, 8] led eventually to the wounded-nucleon picture for hadron- and nucleus-nucleus collisions. The formulas for nuclei cross sections existing in the literature under the name of "Glauber" (or "optical", "eikonal"), are sometimes called "multiple diffraction" or "multiple scattering". They are not always the same, the chain of adopted approximations connects one with the others. Some of them could be questioned, so we would like to examine them briefly below.

\section{1 proton-nucleus case}

The nucleus consisting of $A$ nucleons can be seen by incoming high energy nucleon (by projectile) as a system of independent scattering centers. The essence of the Glauber approximation 


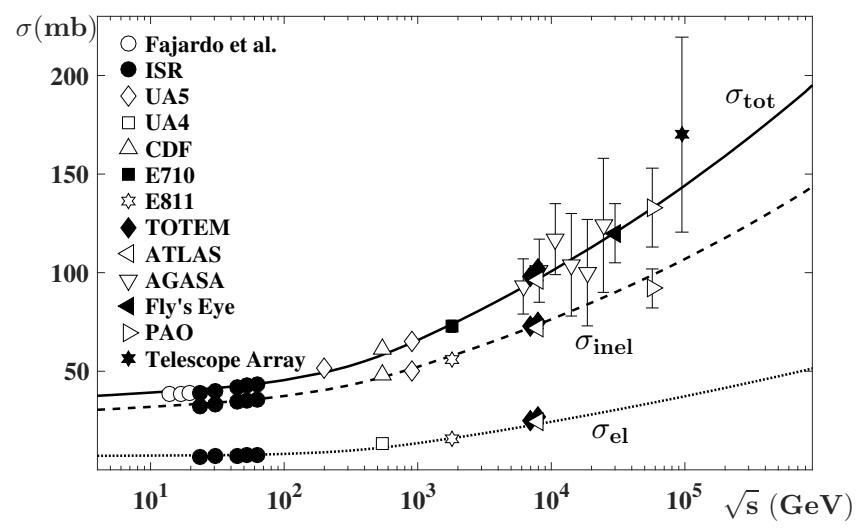

Figure 2: Values of the elastic, inelastic and total cross section calculated with optical model as a function of the interaction en- ergy compared with the measurements. Solid line represents total cross section, dashed inelastic and dotted line elastic cross sec- tion predictions. Points are experimental data.

is the natural assumption that the resulting amplitude phase shift of the collision is the sum of all possible $A$ individual nucleon-nucleon phase shifts

$$
\chi_{A}(b,\{\mathbf{d}\})=\sum_{j=1}^{A} \chi\left(\mathbf{b}-\mathbf{d}_{j}\right)
$$

where $\{\mathbf{d}\}$ is a set of nucleon positions in the nucleus $\left(\mathbf{d}_{j}\right.$ is the position of the $j$ th nucleon in the plane perpendicular to the interaction axis). The scattering amplitude is

$$
S(t)=\frac{i}{2 \pi} \int \mathrm{e}^{i \mathbf{t b}} d^{2} \mathbf{b} \int|\varphi(\{\mathbf{d}\})|^{2}\left\{1-\mathrm{e}^{i \chi_{A}(b,\{\mathbf{d}\})}\right\} \prod_{j=1}^{A} d^{2} \mathbf{d}_{j}
$$

where the function $\varphi$ is the wave-function of the nucleus with nucleons distribution given by the $\{\mathbf{d}\}$. This general formula is a subject of subsequent approximations leading to the set of consecutively simpler equation for the collision cross sections. First we assume no space correlation between nucleons. With the universal nucleon distribution within the nucleus $\rho$ we obtain

$$
|\varphi(\{\mathbf{d}\})|^{2}=\prod_{j=1}^{A} \rho_{j}\left(\mathbf{d}_{j}\right)
$$

with the following normalization: $\left(\int \rho_{j}\left(\mathbf{r}_{j}\right) d^{3} \mathbf{r}_{j}=1\right)$.

Next quite obvious approximation is that individual sub-collisions are the same, having universal nucleon-nucleon phase-shifts dependences $\chi$. With this we have

$$
\begin{array}{r}
S(t)=\frac{i}{2 \pi} \int \mathrm{e}^{i \mathbf{t b}} d^{2} \mathbf{b} \int \prod_{j=1}^{A} \rho_{j}\left(\mathbf{d}_{j}\right)\left\{1-\mathrm{e}^{i \sum_{j=1}^{A} \chi\left(\mathbf{b}-\mathbf{d}_{j}\right)}\right\} d^{2} \mathbf{d}_{j}= \\
=\frac{i}{2 \pi} \int \mathrm{e}^{i \mathbf{t b}} d^{2} \mathbf{b}\left\{1-\int \prod_{j=1}^{A} \rho_{j}\left(\mathbf{d}_{j}\right) \mathrm{e}^{i \chi\left(\mathbf{b}-\mathbf{d}_{j}\right)} d^{2} \mathbf{d}_{j}\right\}
\end{array}
$$


On the other hand, the scattering process can be treated as the single collision process with its own nuclear phase shift $\chi_{\mathrm{opt}}(b)$

$$
S(t)=\frac{i}{2 \pi} \int \mathrm{e}^{i \mathbf{t b}}\left\{1-\mathrm{e}^{i \chi_{\mathrm{opt}}(b)}\right\} d^{2} \mathbf{b}
$$

The comparison of Eqs.(3.4) and (3.5) leads to the relation between the opacity for the nucleus and the single nucleon:

$$
\mathrm{e}^{i \chi_{\mathrm{opt}}(b)}=\int|\varphi(\{\mathbf{d}\})|^{2} \mathrm{e}^{i \sum_{j=1}^{A} \chi_{j}\left(\mathbf{b}-\mathbf{d}_{j}\right)} d^{2} \mathbf{d}_{j}=\left\langle\mathrm{e}^{i \chi(b,\{\mathbf{d}\})}\right\rangle
$$

\subsection{Big nucleus, point nucleon approximation}

The calculation of the integral in Eq.(3.6) requires the knowledge of the fluctuation of the nucleus shape. Some interesting effects relating to this are discussed in $[10,11]$, but for our averaging integral, we assume that the nucleus with a given number of constituent nucleons $A$ is always the same. The shape of the 'average nucleus 'we adopted from the Lund model as described in [12] is in the form of Woods-Saxon distribution:

$$
\rho_{A}(d)=\frac{\rho_{0}}{1+\exp \left(\frac{d-d_{0}}{\delta}\right)}
$$

(for nuclei heavier than oxygen; the Gaussian for lighter nuclei) with two parameters $\left(d_{0}\right.$ the 'halfdensity' radius) and $\delta$ related to the 'surface thickness' of the nucleus, respectively. For the convenience the density $\varphi$ is normalized to $A$.

If the number of nucleons $(A)$ goes to infinity keeping obviously nucleus opacity constant then

$$
\chi_{\text {opt }}(b)=i \int d^{2} \mathbf{d} \rho_{A}(d)\left(1-\mathrm{e}^{i \chi(\mathbf{b}-\mathbf{d})}\right)
$$

Opacity of the nucleon is the sum of many (infinitely many, but accually $A>6$ should be big enough [2]) of independent nucleons as small (infinitely small, as A goes to infinity) scattering centers.

Using Eq.(2.5) and the optical theorem the integration in Eq.(3.8) can be performed and for the case of pure imaginary scattering amplitude we obtain

$$
\chi_{\mathrm{opt}}(b)=\frac{1}{2} \sigma_{\mathrm{pp}}^{\mathrm{tot}} \rho_{A}(b)
$$

The opacity given by Eq.(3.9) can be substituted to Eq.(2.5) and,e.g., the inelastic cross section could be eventually obtained

$$
\begin{aligned}
& \left.\sigma_{\text {inel }}^{p A}=\int\left[1-\mathrm{e}^{-\sigma_{\mathrm{pp}}^{\mathrm{tot}} \rho_{A}(b)}\right)\right] d^{2} \mathbf{b}, \\
& \quad=\int\left\{1-\left[1-\sigma_{\mathrm{pp}}^{\mathrm{tot}} \frac{\rho_{A}(b)}{A}\right]^{A}\right\} d^{2} \mathbf{b} .
\end{aligned}
$$

Both formulas, Eq.(3.10) and Eq.(3.11) define the so-called "point nucleon" approximation. 


\subsection{Nucleus-nucleus collision}

The treatment of nucleon-nucleus presented above can be extended to the case of nuclei collisions with the amplitude defined as

$$
\begin{array}{r}
S(t)=\frac{i}{2 \pi} \int \mathrm{e}^{i \mathbf{t b}} d^{2} \mathbf{b}\left\{1-\int d^{2} \mathbf{d}_{i} \prod_{i=1}^{A} \rho_{A}\left(\mathbf{d}_{i}\right) \int d^{2} \mathbf{d}_{j} \prod_{j=1}^{B} \rho_{B}\left(\mathbf{d}_{j}\right) \mathrm{e}^{i \chi\left(\mathbf{b}-\mathbf{d}_{i}-\mathbf{d}_{j}\right)}\right\}= \\
=\frac{i}{2 \pi} \int \mathrm{e}^{i \mathbf{t b}} d^{2} \mathbf{b}\left\{1-\int d^{2} \mathbf{d}_{i} \rho_{\mathrm{AB}}\left(\mathbf{d}_{i}\right) \mathrm{e}^{i \chi\left(\mathbf{b}-\mathbf{d}_{i}\right)}\right\}
\end{array}
$$

with

$$
\rho_{\mathrm{AB}}(b)=\int d^{2} \mathbf{d}_{i} \prod_{i=1}^{A} \rho_{A}\left(\mathbf{b}-\mathbf{d}_{i}\right) \int d^{2} \mathbf{d}_{j} \prod_{j=1}^{B} \rho_{B}\left(\mathbf{d}_{j}\right)
$$

Using Eq.(3.5) we can define the overall nucleus-nucleus opacity

$$
\mathrm{e}^{i \chi_{\mathrm{AB}}(b)}=\int d^{2} \mathbf{d}_{i} \rho_{\mathrm{AB}}(\{\mathbf{d}\}) \mathrm{e}^{i \sum_{i=1}^{A} \chi\left(\mathbf{b}-\mathbf{d}_{i}\right)}=\left\langle\mathrm{e}^{i \chi\left(b,\left\{\mathbf{d}_{\mathbf{A}}\right\},\left\{\mathbf{d}_{\mathbf{B}}\right\}\right)}\right\rangle
$$

Thus the nucleus-nucleus scattering amplitude is, with the analogy to Eq.(2.1)

$$
S(\mathbf{t})=\frac{i}{2 \pi} \int \mathrm{e}^{i \mathbf{t b}}\left(1-\mathrm{e}^{i \chi_{A B}(b)}\right) d^{2} \mathbf{b}
$$

The "big nucleus" and "point nucleon" approximation can be used also in this case leading to

$$
\begin{aligned}
\sigma_{\text {inel }}^{A B} & \left.=\int\left[1-\mathrm{e}^{-\sigma_{\mathrm{pp}}^{\mathrm{tot}} \rho_{A B}(b)}\right)\right] d^{2} \mathbf{b}, \\
& =\int\left\{1-\left[1-\sigma_{\mathrm{pp}}^{\mathrm{tot}} \frac{\rho_{A B}(b)}{A B}\right]^{A B}\right\} d^{2} \mathbf{b} .
\end{aligned}
$$

\subsubsection{The probabilistic framework}

The nucleus-nucleus inelastic interactions are the ones defining the development of nuclear cascades of the Extensive Air Showers, and their description is crucial to the interpretation of the cosmic ray data, thus there exist in the literature few more ways of their treatment. Quite popular is the probabilistic formalism (see, e.g., [13]). It assumes that the collision between individual nucleons of colliding nuclei are not correlated and do not interfere with each other. The probability of each (inelastic) interaction works here and it is calculated using the Eq.(2.1) in each subcollision case. The usage of the "big nucleus" and "point nucleon" approximation in the probabilistic framework leads to the inelastic interaction probability with the given impact parameter $b$ [14]

$$
p_{A B}(b)=\frac{\rho_{A B}(b)}{A B} \sigma_{\text {inel }}^{p p}
$$

If there are $A B$ pairs of nucleons which could take part in the interaction the probability of having $n$ inelastic interaction is

$$
p(n, b)=\left(\begin{array}{c}
A B \\
n
\end{array}\right)\left[\frac{\rho_{A B}(b)}{A B} \sigma_{\text {inel }}^{p p}\right]^{n}\left[1-\frac{\rho_{A B}(b)}{A B} \sigma_{\text {inel }}^{p p}\right]^{A B-n},
$$


The summation over $n$ can be performed and the result with the integration over $b$ gives the value of the so-called "production cross section":

$$
\sigma_{\text {inel }}^{A B}=\int d^{2} \mathbf{b}\left\{1-\left[1-\frac{\rho_{A B}(b)}{A B} \sigma_{\text {inel }}^{p p}\right]^{A B}\right\},
$$

which is quite similar to the result in Eq.(3.17), but with $\sigma_{\text {inel }}$ in place of $\sigma_{\text {tot }}$.

\section{Results}

As a final result of this work we present values of p-Air and Fe-Air cross sections calculated using various methods described previously. Figures present energy dependence of elastic, inelastic and total cross sections calculated using exact Glauber model and "point nucleon" aaproximation with $\sigma_{t o t}^{p p}$, and $\sigma_{\text {inel }}^{p p}$ as parameters.

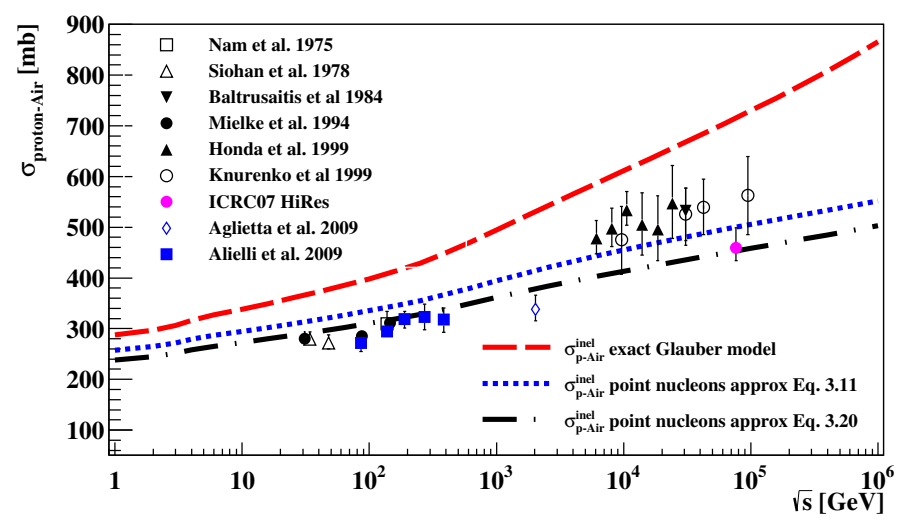

Figure 3: Comparison of $\sigma_{p-A i r}^{\text {inel }}$ calculated with various method with experimental data [15]. Pure Glauber theory - red line, "point nucleon" approximation - blue and black lines
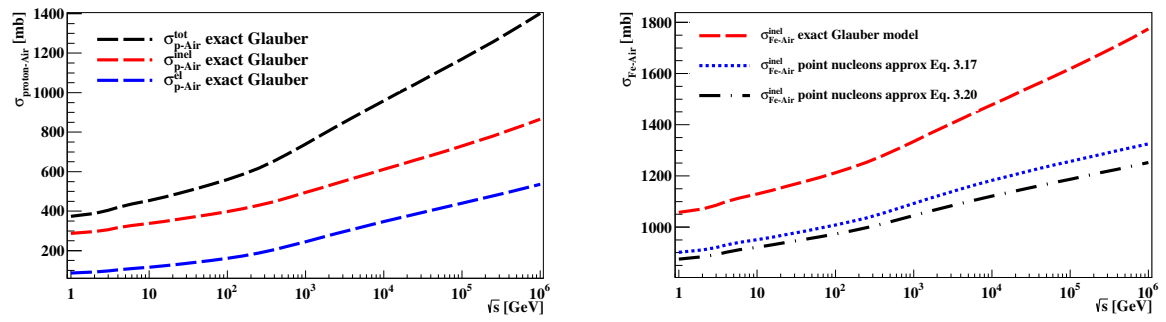

(a) Results of Glauber theory for p-Air colli- (b) Comparison of $\sigma_{\mathrm{Fe}-\mathrm{Air}}^{\text {inel }}$ calculated with sions. Opacity function $\chi(b)$ calculated using Glauber theory (Eq. 3.8, red line) and with Eq. 3.8 "point nucleon" approximation (blue and black lines)

Figure 4: Elastic, inelastic and total cross section for p-Air calculated using Glauber theory (a) and inelastic cross sections for Fe-Air calculated with different methods (b) 


\section{Conclusions}

In this work we present extrapolation in general of nucleus nucleus cross sections up to cosmic ray energies using Glauber model and its modifications. In those calculations we relied on previosly performed the optical model for the description of proton-proton collisions. The comparison of the exact Glauber model and "point nucleon" aproximation are presented in comparison with experimental data for p-Air cross section. Point nucleon aproximation shows better aligment with experimental data. Works on the probabilistic framework are in progres and results will be presented in the future.

Acknowledgments: This work was supported by grant no 2016/20/T/ST9/00589 funded by the National Science Centre in Poland.

\section{References}

[1] R. J. Glauber, Lectures in theoretical physics, Vol. 1., edited by W.E. Brittin et al. (Interscience Publishers, New York, 1959) p. 315.

[2] R. J. Glauber and G. Matthiae, Nuclear Physics B 21, 135, (1970).

[3] R. J. Glauber, Nuclear Physics A 774, 3 (2006), QUARK MATTER 2005.

[4] Z. Plebaniak, T. Wibig, Physics Letters B 761, 469 (2016).

[5] W. Czyż and L. Leśniak, Physics Letters B. 24, 227, (1967).

[6] W. Czyż and L.C. Maximon, Physcis Letters B 27, 354 (1968).

[7] W. Czyż and L.C. Maximon, Annals of Physics, 52, 59 (1969).

[8] A. Białas and W. Czyż, Physics Letters B 51, 179 (1974).

[9] A. Białas, M. Bleszyński, and W. Czyż, Acta Phys. Polon. B8, 389 (1977).

[10] G. Wilk and Z. Włodarczyk, 32nd ICRC, Beijing, (2011)

[11] V. Guzey and M. Strikman, Physics Letters B 633, 245 (2006)

[12] L. Ding and E. Stenlund, Computer Physics Communications, 59, 313 (1990)

[13] J. Engel, T. K. Gaiser, P. Lipari and T. Stanev, Phys Rev. D 46, 5013 (1992)

[14] M. L. Miller, K. Reygers, S. J. Sanders, P. Steinberg, Ann. Rev. Nucl. Part. Sci 57, 205 (2007), arXiv:nucl-ex/0701025[nucl-ex]

[15] R. Ulrich et al. New J.Phys. 11 (2009) 065018 arXiv:0903.0404 [astro-ph.HE] 